\title{
IMPACT OF RAPID EYE MOVEMENT SLEEP DEPRIVATION ON HYPOTHALAMIC-PITUITARY- TESTICULAR AXIS IN WISTAR ALBINO RATS
}

\author{
DHEEPTHI JAYAMURALI, SATHYA NARAYANAN GOVINDARAJULU*
}

Department of Physiology, Dr. ALM Post Graduate Institute of Basic Medical Sciences, University of Madras, Taramani, Chennai 600113, Tamil Nadu, India. Email: drgsathyanarayanan@gmail.com

Received: 10 April 2018, Revised and Accepted: 09 May 2018

\section{ABSTRACT}

Objectives: The objectives of the study were to study the impact of rapid eye movement (REM) sleep deprivation (SD) on the hypothalamic-pituitarytesticular (HPT) axis in the Wistar albino rats.

Methods: Adult male Wistar albino rats weighing about $200 \mathrm{~g}$ were segregated into Group I-IV of control, 48 h SD, 72 h SD, and 96 h SD, respectively, in a custom-made SD tank. After SD procedure, the sexual behavior of rats was assessed, after which blood was collected from the animals for the estimation of HPT and stress hormones and then the testicles were used for histological studies.

Results: SD has increased $(\mathrm{p}<0.05)$ the mounting latency and intromission latency and decreased $(\mathrm{p}<0.05)$ the mounting frequency and intromission frequency compared with control group. A significant increase $(p<0.05)$ in corticosterone, follicle stimulating hormone levels and a significant decrease $(\mathrm{p}<0.05)$ in gonadotropin-releasing hormone, luteinizing hormone and testosterone levels were observed in all the SD groups when compared with control group. SD-induced testis architecture displayed sperm retention, sperm reduction, and shape alteration when compared to the control group. Furthermore, apoptotic bodies were observed in the testis of $72 \mathrm{~h}$ and $96 \mathrm{~h}$ of sleep-deprived animals.

Conclusion: Outcome of the project reveals that out of 48 h, 72 h, and 96 h of SD, 96 h of SD has a great impact on sexual behavior, HPT hormones, and testicular morphology.

Keywords: Rapid eye movement sleep deprivation, Hypothalamic-pituitary-testicular axis, Sexual behavior, Stress, Apoptosis.

(c) 2018 The Authors. Published by Innovare Academic Sciences Pvt Ltd. This is an open access article under the CC BY license (http://creativecommons. org/licenses/by/4. 0/) DOI: http://dx.doi.org/10.22159/ajpcr.2018.v11i8.26635

\section{INTRODUCTION}

World Health Organization (WHO) has reported infertility as a global issue, prevalence, and trends, since 1990 [1]. Sleep disorders are conditions characterized by disruptions of sleep quality or of sleep pattern [2]. It is a type of stress which affects the modern civilization. In general stress-induced variations are self-limiting and adaptive until and unless events that overrule "threshold" bounds become irretrievable and pathological [3]. Far more than 30 million men worldwide were reported to be infertile. Worldwide 48.5 million couples were reported to be affected with primary infertility around 2010 , of which male contribution is involved in $20-70 \%$ of affected couples (WHO, 2012). Male reproductive impairment may result from various factors such as diseases, psychological factors, and hormonal changes that affect sperm production, viability, function, or transport mechanisms. Previous report has arrived to a conclusion that modern lifestyle factors and environmental influences, rather than genetics, are the most vital causal factors of the fertility disorder trends [4]. As evidence continues to accumulate on emerging lifestyle risk factors, such as prolonged sitting and unhealthy sleep patterns [5], integrating these new risk factors to infertility would present clinically significant information on the lifestyle causal aspect of reproductive disorders. Although sleep was considered as a time of rest and recovery from the stresses of everyday life, research has revealed that sleep is a dynamic activity, during which many processes vital to health and well-being take place (National Sleep Foundation, 2006). A recent survey has specified that $93 \%$ Indians are sleep deprived. Sleep deprivation (SD) occurs when insufficient sleep leads to decreased performance, poor alertness, and deterioration in health. SD has various deleterious effects on the body including increased risk of stroke, obesity, diabetes, cancer, permanent cognitive deficits, osteoporosis, cardiovascular diseases, and mortality [6]. Although the integral role of sleep in many chronic illnesses has been increasingly recognized over the past few decades, sleep has an underestimated role in sexual function of the life. Studies have shown that normal sleep is required for the proper development of Hypothalamic-Pituitary-Gonadal (HPG) axis [7]. A recent research has demonstrated that paradoxical sleep loss can promote marked changes in the male reproductive system of rats, particularly affecting spermatic function in part by interfering in the testicular nitric oxide pathway [8]. SD stress is of major concern in the current scenario, and limited printings are available on its impact on male reproductive health. Healthy male reproductive pattern depends on the HPG hormones [9], sexual behavior [10], testis architecture [11], sperm parameters [8], and gene expression associated with spermatogenesis [12].

Previous studies in our department on rapid eye movement (REM) SD of three durations: 48,72 , and $96 \mathrm{~h}$ revealed that the sperm count, viability, and morphology were significantly affected in the $48 \mathrm{~h}, 72 \mathrm{~h}$, and $96 \mathrm{~h}$ of SD when compared to the control group. The present research was designed to determine the impact of REM SD of durations: 48,72 , and $96 \mathrm{~h}$ on the sexual behavior, HPG hormones, testicular apoptosis, and histology of testis.

\section{METHODS}

\section{Preparation of animals}

The experimental procedures described herein were performed in accordance with the Ethical Principles in Animal Research adopted by the Committee for the Purpose of Control and Supervision of Experiments on Animals (CPCSEA). The animals used in this experiment were approved by Institutional Animal Ethical Committee (IAEC) of Institute of Basic Medical Sciences (IBMS), University of Madras, Chennai (IAEC No: 01/42/2015). 
24 adult male Wistar albino rats weighing about $200 \mathrm{~g}$ aged about 12 weeks were used in this study. The animals were bred and raised in the animal house of Institute of Basic Medical Sciences. The animals were housed as a colony in standard cages with normal room temperature and a 12-h/12-h light/dark cycle. The animals had free access to food and water - ad libitum. Healthy and matured male albino rats showing dynamic sexual activity were selected for the study because sexually inexperienced male rats can exhibit low performance. Animals were divided into four groups: Control group, $48 \mathrm{~h}$ of SD, $72 \mathrm{~h}$ of SD, and 96 $\mathrm{h}$ of SD. Six animals per group were used in this study. Control animals were maintained in the same tank with the same setup but without water.

\section{REMSD}

Sexually brisk rats were subjected REMSD by modified multiple platform method (MMPM) [13]. MMPM consisted of a tilted water tank with 6 circular platforms of $6.5 \mathrm{~cm}$ diameter. The tanks were filled with $1 \mathrm{~cm}$ of water from the bottom of the tank. The rats were placed individually on top of each platform so that the rats could move within the tank by jumping from one platform to another. Each group was placed in one tank. The animals remained inside the tank for $48 \mathrm{~h}, 72 \mathrm{~h}$, and $96 \mathrm{~h}$, respectively. When the rat enters the REM sleep, it loses its postural control due to decrease in muscle tone. As a result, the rats would touch the surrounding water, and REM sleep will be interrupted. The water in the tank was changed daily throughout the experimental period.

\section{Sexual behavior}

After the $48 \mathrm{~h}, 72 \mathrm{~h}$, and $96 \mathrm{~h}$ of SD protocol, sexual behavior of the rats was evaluated, respectively. The test was carried out in a rectangular arena. Animals from each group were allowed to mate with sexually receptive, and estrus phased females. The testing of sexual behavior was performed under red light illumination during the dark phase of the light/dark cycle. Male sexual behavior was assessed by placing the male in the arena, 5 min before a sexually receptive female was presented and then the test lasted for 30 minutes. The following sexual parameters were recorded: Mounting latency (ML), mounting frequency (MF), intromission latency (IL), and intromission frequency (IF) [14]. The values of the observed parameters for control and experimental groups were recorded. An animal can make several intromissions in a single mount.

\section{Blood sampling and hormone determination}

Immediately after evaluation of the sexual behavior, blood was collected from retro-orbital venous plexus of all the animals before sacrifice. The blood samples were centrifuged for the collection of serum and were stored frozen at $-20^{\circ} \mathrm{C}$ until use. Samples were assessed for stress hormone - corticosterone (CORT) and reproductive hormones - gonadotropin-releasing hormone $(\mathrm{GnRH})$, luteinizing hormone $(\mathrm{LH})$, follicle stimulating hormone (FSH), and testosterone (T) by Enzymelinked immune sorbent assay kit (CUSABIO, USA).

\section{Histological analysis}

Studies had indicated that testicular dysfunctions are associated with histological variations. Two parallel slices perpendicular to the testicular long axis were cut from each testis, and half of the each slice was cut for embedding. Testes after removal was immersed in paraformaldehyde fixation and processed until embedded in paraffin for histological analysis. $5 \mu$ thick sections were prepared using microtome. The sections were stained with standard Hematoxylin and Eosin method for histomorphological analyses of the testes. The sections were also stained with modified trichrome technique for the detection of apoptotic bodies since apoptosis occurs under many pathological conditions.

\section{Statistical analysis}

All data were analyzed using SPSS (version 20.0). Data are expressed as line diagram and bar diagram with Mean \pm SD. Statistical significance between the different groups was determined by one-way analysis of variance followed by Tukey's multiple comparison tests. $\mathrm{p}<0.05$ was considered significant.

\section{RESULTS}

\section{Sexual behavior}

SD has significantly altered the sexual behavioral parameters of the animals when compared with control group. Significant alterations were found in the mounting parameters. All the sleep-deprived groups displayed a significant increase in the ML when compared with the control group. Moreover, $96 \mathrm{~h}$ of sleep-deprived animals showed a significant increase $(\mathrm{p}<0.05)$ in the ML when compared with $48 \mathrm{~h}$ (Fig. 1). $72 \mathrm{~h}$ and $96 \mathrm{~h}$ SD animals exhibited a significant decrease $(p<0.05)$ while $48 \mathrm{~h}$ of SD presented no significant alterations in the MF when compared with the control group (Fig. 2).

The sleep-deprived groups presented significant alterations in the intromission parameters when compared with control group. All the sleep-deprived groups had a longer latency for the first intromission when compared with the control groups. $72 \mathrm{~h}$ and $96 \mathrm{~h}$ of SD animals showed a significant increase $(\mathrm{p}<0.05)$ in the IL when compared with $48 \mathrm{~h}$ of SD (Fig. 3). A significant decrease $(\mathrm{p}<0.05)$ in the IF was observed in $72 \mathrm{~h}$ and $96 \mathrm{~h}$ of sleep-deprived groups when compared to the control and $48 \mathrm{~h}$ sleep-deprived animals (Fig. 2).

\section{Impact of REMSD on stress hormone}

As a confirmation of SD stress, the hormonal profile of CORT was detected and perhaps it revealed significant alterations among the four groups. The hormonal level of CORT was significantly increased $(p<0.05)$ in all the sleep-deprived groups when compared with the control group (Fig. 4)

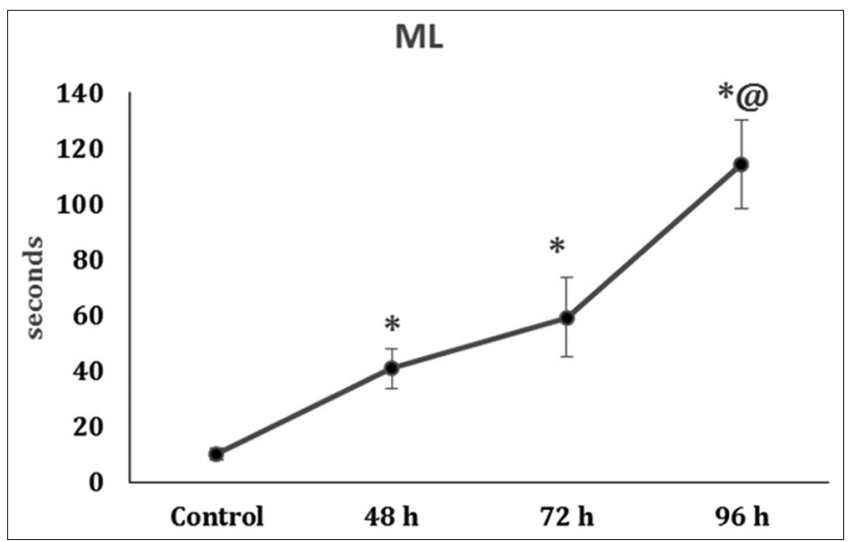

Fig. 1: Mounting latency. *Indicates significance when compared with control. ${ }^{\circledR}$ Indicates significance when compared with $48 \mathrm{~h}$ of sleep-deprived animals. $\mathbf{p}<0.05$ is considered significant

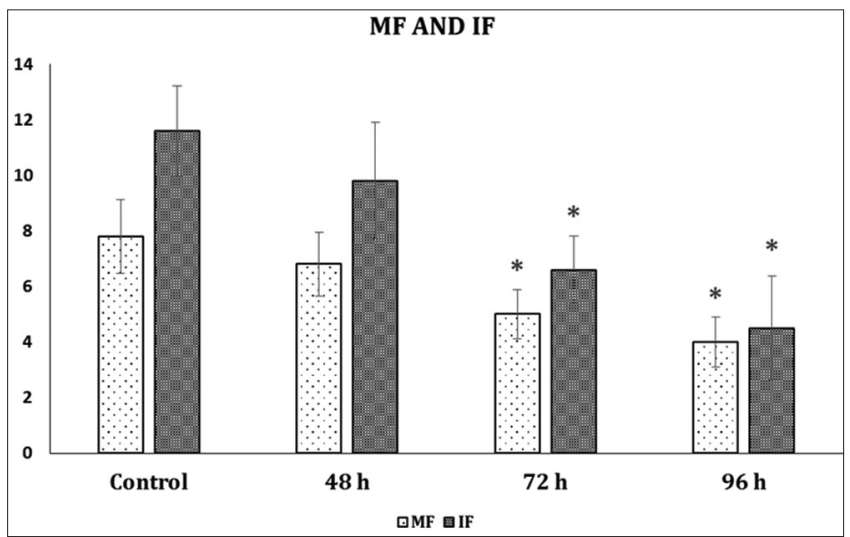

Fig. 2: Mounting frequency and intromission frequency. *Indicates significance when compared with control. $p<0.05$ is considered significant 
Impact of REMSD on reproductive hormones

As a confirmation for REM, SD-induced alterations in the hypothalamicpituitary-testicular axis, the hormones concerned with HPG axis were estimated. Substantial alterations of hormonal profiles were observed in SD groups when compared with the control group. A significant decrease $(p<0.05)$ in the levels of GnRH, LH, and T (Figs. 5-7) was observed in all the sleep-deprived groups when compared with the control groups. The hormonal levels of FSH presented a significant increase $(\mathrm{p}<0.05)$ in the $72 \mathrm{~h}$ and $96 \mathrm{~h}$ of sleep-deprived groups when compared with the control and $48 \mathrm{~h}$ of SD groups (Fig. 8).

\section{Histological outcomes}

Testicular histology of control rats displayed normal and undisturbed pattern in the arrangement and shape of seminiferous tubules

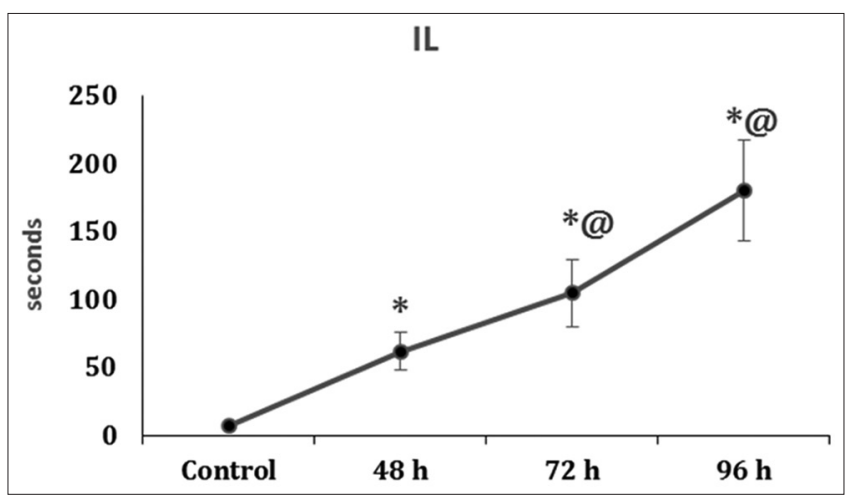

Fig. 3: Intromission latency. *Indicates significance when compared with control. @Indicates significance when compared to $48 \mathrm{~h}$ sleep-deprived animals. $\mathrm{p}<\mathbf{0 . 0 5}$ is considered significant

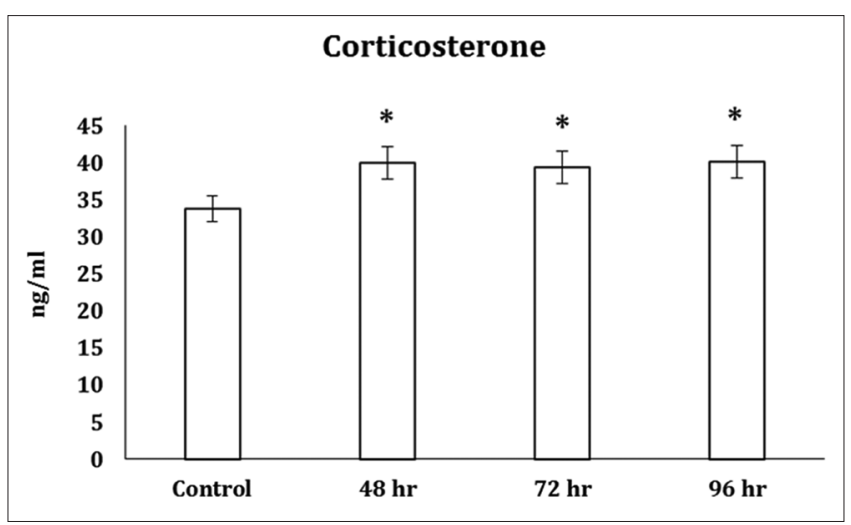

Fig. 4: Serum corticosterone level. *Indicates significance when compared with control. $\mathrm{p}<\mathbf{0 . 0 5}$ is considered significant

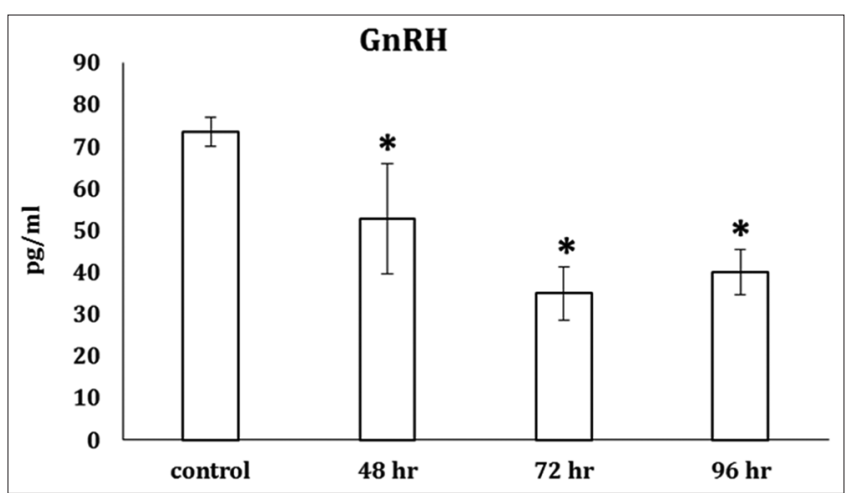

Fig. 5: Serum gonadotropin-releasing hormone level. *Indicates significance when compared with control. $p<0.05$ is considered significant
(Fig. 9a). Microscopic analysis of testicular histology of SD rats under $400 \times$ magnifications showed variations in the shape of the tubular arrangement when compared with the control group. Chiefly, the $72 \mathrm{~h}$ and $96 \mathrm{~h}$ of SD group animals displayed oval tubular like shape (Fig. 9c and d). Sperm retention was visibly observed in the SD groups when compared to the control group (Fig. 9b-d). Disturbed spermatogonia and Sertoli cell pattern were observed in the $72 \mathrm{~h}$ and $96 \mathrm{~h}$ of sleep-deprived animals in comparison to the control animals (Fig. 9c and d).

To quantify the number of apoptotic cells in the sleep-deprived testis, the modified trichrome staining method was used in this study and SD exhibited marked changes at the cellular level of the testis. Normal cells appeared to be green in color. The apoptotic cells stained to be dark

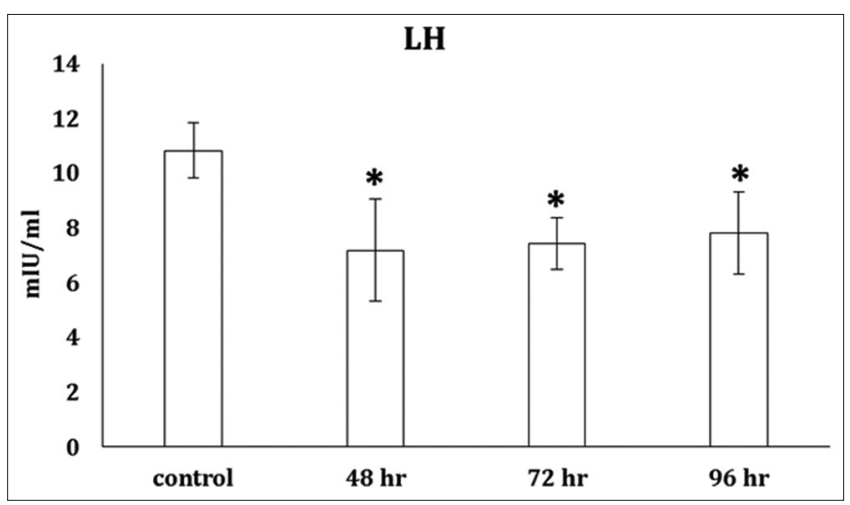

Fig. 6: Serum luteinizing hormone level. *Indicates significance when compared with control. $p<0.05$ is considered significant

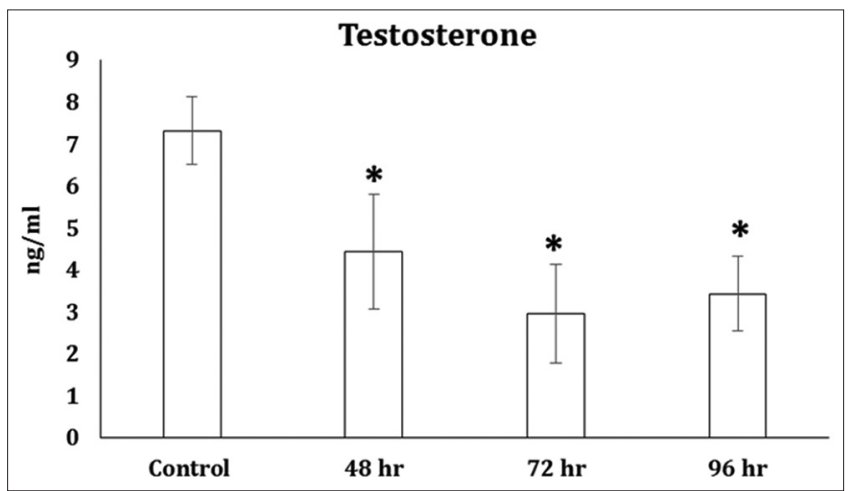

Fig. 7: Serum testosterone level. *Indicates significance when compared with control. $\mathrm{p}<0.05$ is considered significant

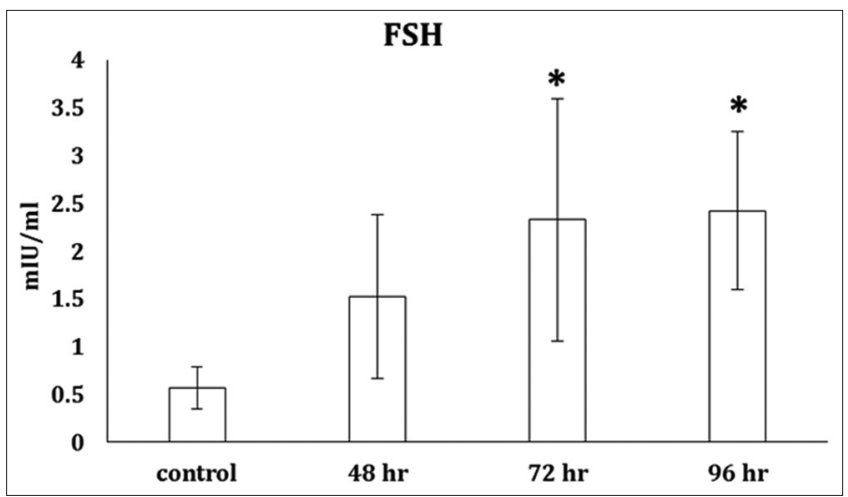

Fig. 8: Serum follicle stimulating hormone level. *Indicates significance when compared with control. $p<0.05$ is considered significant 
red in color. The control group was observed to be completely stained green in color (Fig. 10a). Testicular cells of $72 \mathrm{~h} \mathrm{SD}$ animals displayed rare apoptotic bodies (Fig. 10c). Many dark red stained cells were witnessed in the testis of $96 \mathrm{~h}$ of SD animals, indicating an increased rate of apoptosis (Fig. 10d).

Arrow indicates apoptotic bodies in brown color.

\section{DISCUSSION}

In the present study, SD inhibited the sexual behavior in the male rats. This finding goes in parallel with previous literature which represents lower sexual performance such as increased latency to mount and initiate intromission, decreased intromission and MF in the $96 \mathrm{~h}$ of paradoxical SD animals [8]. It has been previously reported that total SD decreases the $\mathrm{T}$ levels $[15,16]$. There is evidence that $\mathrm{T}$ activates the sexual behavior in rodents [17]. Our study also reveals decreased $\mathrm{T}$

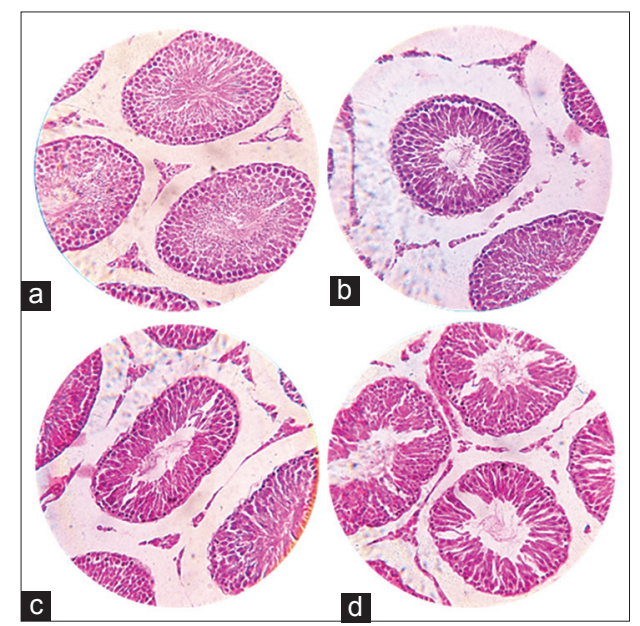

Fig. 9: Hematoxylin and Eosin staining of testes under 400x magnification. (a) Control (b) $48 \mathrm{~h}$ sleep deprivation (SD) (c) $72 \mathrm{~h}$ SD (d) $96 \mathrm{~h} \mathrm{SD}$

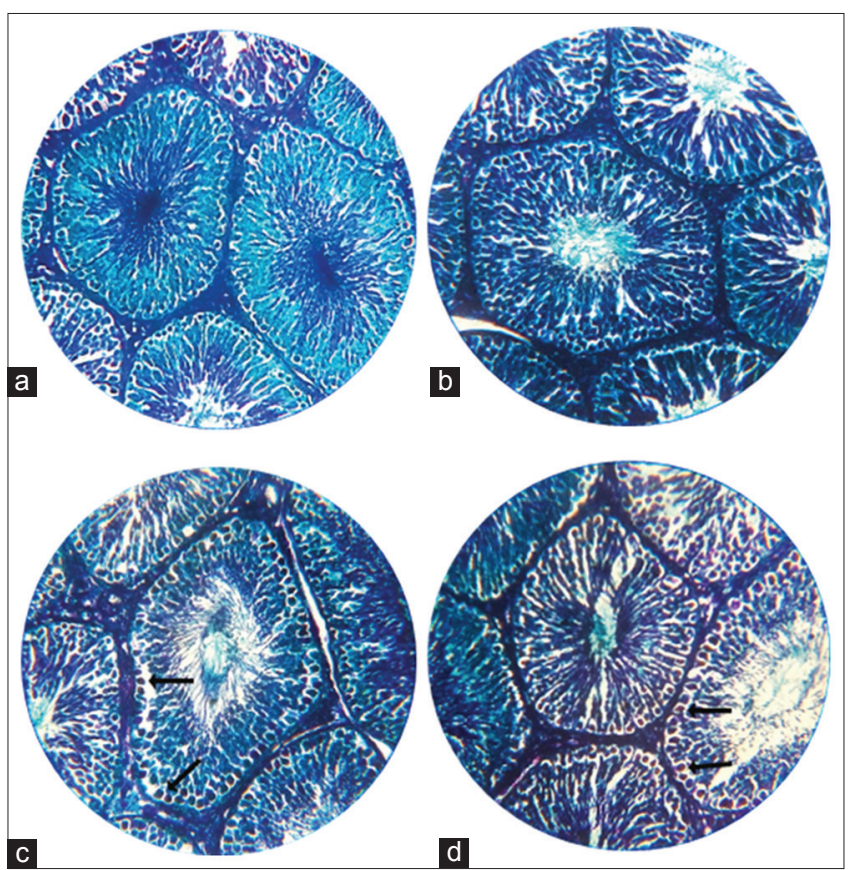

Fig. 10: Modified trichrome staining of testes under 400× magnification. (a) Control (b) $48 \mathrm{~h}$ sleep deprivation (SD) (c) $72 \mathrm{~h}$ SD (d) $96 \mathrm{~h}$ SD hormonal profile after SD. This may contribute, at least in part, with the changes in the sexual behavioral parameters.

In the present study, we also proposed to evaluate the HPG hormones that could help us clarify mechanisms underlying decreased sexual behavior after lack of sleep. Any stressful stimuli, particularly SD in the current study might have increased the CORT levels. Furthermore, other research studies support the fact that SD elevates the plasma CORT levels [18]. Although there is evidence that stress hormone CORT inhibits the gonadal function at multiple levels in hypothalamic-pituitary axis: (1) At the level of hypothalamus, it inhibits GnRH $[19,20]$ and (2) at the level of pituitary, to decline LH [21] and FSH secretions [22], this is not always followed by FSH reduction [23] as observed in this study. In the present study as expected, the levels of $\mathrm{T}$ were greatly decreased. The inhibition of the T may be due to the direct action of the glucocorticoid CORT on the testis [24]. Review studies on glucocorticoids and infertility have reported that glucocorticoid receptor (GR) is present in the multiple interstitial cell types of the testis, including Leydig cell, macrophages, and epididymis [25]. These findings support the direct action of CORT on the testis and contribute to the drop in the level of T.

Any detrimental effect of SD on male reproduction was visibly confirmed by abnormal testicular histopathology. In the current study, a change in the tubular shape was observed in the sleep-deprived group. This is underscored from previous conclusions which displayed that hormonal imbalance caused the shape of the tubules to become an oval-like shape. This change in the tubular shape leads to the elongation of the tubules [26]. In the present study, $48 \mathrm{~h}, 72 \mathrm{~h}$, and $96 \mathrm{~h} \mathrm{SD}$ groups showed decreased sperm count. This may be due to the direct effect of CORT on the testis or may be due to hormonal imbalances. Previous studies in our department have established the fact that sperm count decreases after SD protocol which is confirmed visibly in the current study. Furthermore, sperm retention and atrophy in the seminiferous tubules of SD animals observed in the current study goes similar to previous literature which displayed sperm retention in $96 \mathrm{~h} \mathrm{SD}$ group [27].

There is evidence that SD induces Leydig cell apoptosis by caspase 3 activation through the elevation of CORT [28]. Therefore, in the current study, the apoptotic cells observed in the testis of $72 \mathrm{~h}$ and $96 \mathrm{~h}$ of sleepdeprived animals might be due to the activation of caspase 3 after SD.

In summary, we have presented evidence that exposure to SD has well-known roles in affecting the HPG axis in the male rats. The actual mechanism underlying gonadal dysfunction in the present study includes hormonal imbalance and the consequent alterations in the sexual behavior; marked histological changes in the testis architecture; SD-induced apoptosis in the testicular cells. Further, studies on the herbal treatment of SD can provide a remedy for the pathology and may enhance the reproductive health.

\section{ACKNOWLEDGMENT}

The authors thank the University Grants Commission (No.F.30-93/2015 BSR) for providing the financial support.

\section{AUTHOR'S CONTRIBUTION}

All the authors have contributed equally.

\section{CONFLICTS OF INTEREST}

Authors declare that they have no conflicts of interest.

\section{REFERENCES}

1. Mascarenhas MN, Flaxman SR, Boerma T, Vanderpoel S, Stevens GA. National, regional, and global trends in infertility prevalence since 1990: A systematic analysis of 277 health surveys. PLoS Med 2012;9:e1001356.

2. Manjula T, Shakti S, Sachin BA. Development and evaluation of sublingual tablet of Zolpidem tartrate an antipsychotic drug. Int $\mathrm{J}$ 
Pharm Pharm Sci 2016;8:179-87.

3. Malathi S, Vidyashree, Ravindran R. Role of Michelia champaca in memory enhancement and acute noise stressed male Wistar albino rats. Int J Pharm Pharm Sci 2016;10:129-35.

4. Skakkebaek NE, Rajpert-De Meyts E, Buck Louis GM, Toppari J, Andersson M, Eisenberg ML, et al. Male reproductive disorders and fertility trends: Influences of environment and genetic susceptibility. Physiol Rev 2016;96:55-97.

5. Ding D, Rogers K, Van der Ploeg H, Stamatakis E, Bauman AE. Traditional and emerging lifestyle risk behaviors and all-cause mortality in middle-aged and older adults: Evidence from a large populationbased Australian Cohort. PLoS Med 2015;12:e1001917.

6. Abrams RM. MD Sleep deprivation. Obstet Gynecol Clin N Am 2015;42:493-506.

7. Hammoud AO, Walker JM, Gibson M, Cloward TV, Hunt SC, Kolotkin RL, et al. Sleep apnea, reproductive hormones and quality of sexual life in severely obese men. Obesity 2011;19:1118-23.

8. Tathiana A, Camila H, Mazaro CR, Tufik Monica LS. Impairment of male reproductive function after sleep deprivation. Fertil Steril 2015; 103:1355-62

9. Saleela M, Robert I, Sarah J. Hormonal regulation of male germ cell development. J Endocrinol 2010;205:117-31.

10. Dixon-Mueller R. The sexuality connection in reproductive health. Stud Fam Plann 1993;24:269-82.

11. Manuela S, Ilpo H. In: Lenzi A, Emmanuele AJ, editors. Endocrinology of the Testis and Male Reproduction. $1^{\text {st }}$ ed. New York: Springer; 2017. p. $273-311$.

12. James A, Miles F. Gene regulation in spermatogenesis. Curr Top Dev Biol 2005;71:131-97.

13. Deborah S, Sergio T. Social stability attenuates the stress in the modified multiple platform method for paradoxical sleep deprivation in the rat. Physiol Behav 2000;68:309-16.

14. Agmo A. Male rat sexual behavior. Brain Res Prot 1997;1:203-9.

15. Schmid SM, Hallschmid M, Jauch-Chara K, Lehnert H, Schultes B. Sleep timing may modulate the effect of sleep loss on testosterone. Clin Endocrinol (Oxf) 2012;77:749-54.

16. Jauch-Chara K, Schmid SM, Hallschmid M, Oltmanns KM, Schultes B. Pituitary-gonadal and pituitary-thyroid axis hormone concentrations before and during a hypoglycemic clamp after sleep deprivation in healthy men. PLoS One 2013;8:e54209.

17. Hull E, Juan M. Sexual behavior in male rodents. Horm Behav 2007;52:45-55.

18. Ilana S, Norman F, Sheila B, Christelle P, Daniel P, Craig H, et al. Sleep deprivation elevates plasma corticosterone levels in neonatal rats. Neurosci Lett 2001;315:29-32.

19. Dubey AK, Plant TM. A suppression of gonadotropin secretion by cortisol in castrated male rhesus monkeys (Macaca mulatta) mediated by the interruption of hypothalamic gonadotropin-releasing hormone release. Biol Reprod 1985;33:423-31.

20. Kamel F, Kubajak CL. Modulation of gonadotropin secretion by corticosterone: Interaction with gonadal steroids and mechanism of action. Endocrinology 1987;121:561-8.

21. Briski KP, Sylvester PW. Acute inhibition of pituitary LH release in the male rat by the glucocorticoid agonist decadron phosphate. Neuroendocrinology 1991;54:313-20.

22. Shannon W, John A. Glucocorticoids, stress, and fertility. Endocrinology 2010;35:109-25

23. Varykina G, Shauna M, Pamela L. Androgens, progestins, and glucocorticoids induce follicle stimulating hormone $\beta$-subunit gene expression at the level of the gonadotrope. Mol Endocrinol 2006;20:2062-79.

24. Bambino TH, Hsueh AJ. Direct inhibitory effect of glucocorticoids upon testicular luteinizing hormone receptor and steroidogenesis in vivo and in vitro. Endocrinology 1981;108:2142-8.

25. Schultz R, Isola J, Parvinen M, Honkaniemi J, Wikström AC, Gustafsson JA, et al. Localization of the glucocorticoid receptor in testis and accessory sexual organs of male rat. Mol Cell Endocrinol 1993;95:115-20.

26. Siti S, Gurmeet K, Aishah M, Mashani M, Vasudevan M, Siti N. Histological changes in testes of rats treated with testosterone, nandrolone, and stanozolol. Iran J Reprod Med 2013;11:653-8.

27. Choi JH, Seung H, Hyun BJ, Sung SJ, Hong S, Young S, et al. Effect of sleep deprivation on the male reproductive system in rats. J Korean Med Sci 2016;31:1624-30.

28. Arjadi F, Partadireja G, Setyawan M, Pangestu M. Paradoxical sleep deprivation changes testicular malondialdehyde and caspase-3 expression in male rats. Univ Med 2015;34:87-95. 